\title{
AN EXAMINATION OF ASSESSOR VARIATION DURING SPORE COUNTING ON SPORE TRAPS OF NEONECTRIA FUCKELIANA
}

\author{
A.J.M. HOPKINS and M.A. DICK \\ Scion, Private Bag 3010, Rotorua, New Zealand \\ Corresponding author: Anna.Hopkins@scionresearch.com
}

\begin{abstract}
In an investigation of the forest fungus Neonectria fuckeliana, ascospores from the air were trapped on petroleum jelly-coated glass slides that were replaced weekly for a year. To determine variation in spore counts between assessors examining the spore traps, four assessors counted the same ten spore traps and spore numbers were compared. Results were highly variable between assessors with almost 100 ascospores (54\% of the higher count) being the difference between the highest and lowest counts for a single trap in one extreme case. To explore sources of assessor variation, the four assessors also counted the same five single transects of a spore trap and results were still highly variable. Two possible sources of variation identified were poor visualisation of ascospores and misidentification of ascospores. Potential solutions to assessor variability include further training, using multiple assessors for each trap and calibrating assessors. Molecular techniques could also be used to quantify spore numbers. Keywords: spore dispersal, Neonectria fuckeliana, spore trapping.
\end{abstract}

\section{INTRODUCTION}

The trapping of spores from the air is a common method for determining the quantity and/or type of fungal spores in the environment during a given period of time (Ingold 1953). It can be used, for example, to sample an airborne spore population to determine the range of species present or to quantify the number of spores of a particular species of interest (e.g. Amsalem et al. 2006). These data can then used to understand the means of spore dissemination and the epidemiology of the fungus (e.g. Geagea et al. 2000). For fungal plant pathogens, this information can be used in the formation of management methods, especially in relation to seasonality and environmental conditions (e.g. Greer \& Webster 2001).

Where data are being used quantitatively for comparison over time and against environmental variables, it is important that spore numbers are recorded accurately. Most counting methods used for environmental samples have errors associated with them and Elphick (2008) broadly classifies these into three groups. First are errors due to portions of the population of interest being unavailable for detection. This can occur either when the target population is not sampled fully or when individuals in the population of interest are present but not detectable, e.g. spores hidden beneath each other. The second type of error is due to detection mistakes. This is when individuals in a population are detectable but are missed for some reason or when non-existent individuals are recorded. The third type of error is due to erroneous counts (double-counting, groups miscounted, recording errors) and includes misidentification of individuals (Elphick 2008). During spore counting all three of these scenarios are possible, particularly where spore traps contain more than one type of fungal spore as well as plant and insect debris.

During an investigation of the epidemiology of the forest fungus Neonectria fuckeliana, a spore trapping programme was undertaken to determine the influence of weather conditions on the dispersal of ascospores. Simple spore traps, consisting of microscope 
slides coated with white petroleum jelly, were changed weekly, resulting in the collection of 312 slides (P.E. Crane, Scion, unpubl. data). These slides were assessed microscopically for ascospores, a process that required clear differentiation of $N$. fuckeliana ascospores from spores of other fungi and from pollen. The time taken to examine a trap varied from 1-3 $\mathrm{h}$ and depended on the amount of biological material deposited on the traps. Due to the time-consuming and repetitive nature of this manual assessment, there were four different assessors. It was thought prudent to examine the reliability of the spore trap data being collected by comparing counts between assessors. This paper outlines the findings of this calibration exercise and discusses the most appropriate way of treating the data given their level of reliability.

\section{METHODS}

\section{The fungus}

Neonectria fuckeliana produces perithecia on the stems of Pinus radiata in parts of New Zealand (Dick \& Crane 2009), and its primary mode of dispersal is thought to be through ascospores (Vasiliauskas \& Stenlid 1997). The ascospores are sticky, and are released and dispersed under moist conditions, either by rain or mist (P.E. Crane, Scion, unpubl. data). Ascospores are hyaline, 14-18 × 6-7 $\mu \mathrm{m}$ and are characterised by a single septum with two equal-sized cells and clear guttules in each cell (Gadgil et al. 2003).

\section{Spore traps}

Spore trapping was carried out in a plantation forest in Southland. Spore traps were microscope slides coated with a smooth layer of melted white petroleum jelly, prepared according to a procedure by Ostry \& Nicholls (1982). They were mounted on a wooden stake with a bull dog clip and were level with, or below clusters of perithecia. Three slides were set up within $1 \mathrm{~m}$ of each of two infected trees that were producing multiple perithecia of $N$. fuckeliana. Slides were collected each week and stored in a refrigerator for later examination. The process of weekly collection of a slide and replacement with a fresh slide was carried out for 12 months with a total of 312 slides collected.

\section{Counting the spores}

In order to determine the number of $N$. fuckeliana ascospores deposited on each slide, the petroleum jelly layer was stained with lactophenol-cotton blue and covered with a large coverslip $(22 \times 50 \mathrm{~mm})$. To maintain accuracy of spore numbers, counting was restricted to within a marked area on the slide that covered $550 \mathrm{~mm}^{2}$. The spores were counted along a transect by scrolling across the slide at $200 \times$ magnification and counting all $N$. fuckeliana ascospores in the field of view. This was done 5 times per slide, which counted the spores in just half of the marked area of the cover slip $\left(275 \mathrm{~mm}^{2}\right)$. If necessary, spore identity was verified at 400× magnification. For each slide, total $N$. fuckeliana ascospore number was recorded, as well as the number of $N$. fuckeliana ascospores found as singles (singletons) and in groups and the size of each group. An ascospore was considered to be in a group if it was less than approximately $5 \mu \mathrm{m}$ away from of another $N$. fuckeliana ascospore.

Due to the large number of slides collected and the time taken to assess each slide (2-3 hours), at least four different assessors were used to count spore traps over the trapping period. Prior to initial commencement of counting, each assessor was shown a slide of ascospores of $N$. fuckeliana extracted directly from perithecia. This allowed them to determine the potential range of variation in size and shape of the ascospores and gave the assessors the best chance of distinguishing $N$. fuckeliana ascospores from other spores and pollen on the slides. In addition, assessors were given access to black and white microscope photographs of $N$. fuckeliana ascospores on slides to use as a reference during counting.

\section{Calibration of spore counting}

A calibration exercise was undertaken to determine the variation in counts between assessors. Ten slides, all of which had been stained and counted previously, were chosen to reflect the range of variation of $N$. fuckeliana ascospore numbers between traps. Four assessors (herein referred to as assessors A to D) counted each slide under the microscope 
as described above. The total number of $N$. fuckeliana ascospores, the number of singletons, the number of ascospores in groups and the size of groups were all recorded. Each assessor independently selected the five transects that were counted. All assessors had significant previous experience counting $N$. fuckeliana ascospores on slides.

\section{Examining single transects}

In order to examine the sources of assessor variation more closely, each of the four assessors also counted the exact same five transects on one slide. This was carried out simultaneously with the main calibration exercise. Each transect was undertaken individually with assessors counting one after another to make sure that the same fields of view were seen. Numbers of $N$. fuckeliana ascospores were recorded as totals, singletons and groups as for the calibration.

\section{Statistical analysis}

For both the main calibration and the examination of single transects, differences in counts were examined statistically to determine whether some assessors were consistently different to all others. For all analyses, each assessor's counts for all traps were pooled and the difference between assessors analysed using a one-way ANOVA. Total $N$. fuckeliana ascospore numbers were examined as well as the difference in counting of singleton and grouped ascospores.

\section{Calibration of spore counting}

\section{RESULTS}

None of the four assessors recorded any $N$. fuckeliana ascospores on slides 1 and 2 (Fig. 1) and these slides were excluded from further analyses. Significant differences between assessors in total ascospore number were recorded for the remaining eight slides counted $(\mathrm{P}<0.0001)$. These differences in ascospore numbers were highly variable between slides and between assessors, but some overall patterns emerged. Assessor A counted significantly more ascospores $(83 \pm 5$ spores/slide $)$ over the eight slides than any other assessor and Assessor D counted significantly fewer ascospores ( $51 \pm 4$ spores/slide) than any other assessor. Assessors B ( $61 \pm 4$ spores/slide) and C ( $73 \pm 5$ spores/slide) did not differ in the number of spores counted. These differences between assessors were reflected in the results of the analysis of singleton ascospores $(\mathrm{P}<0.0001)$, but for counts of grouped ascospores no significant difference was found between assessors $(\mathrm{P}=0.0505)$. Grouped ascospores accounted for an average of $45 \%$ of the total spores counted, although this was highly variable between assessors and spores traps (range 7-90\%).

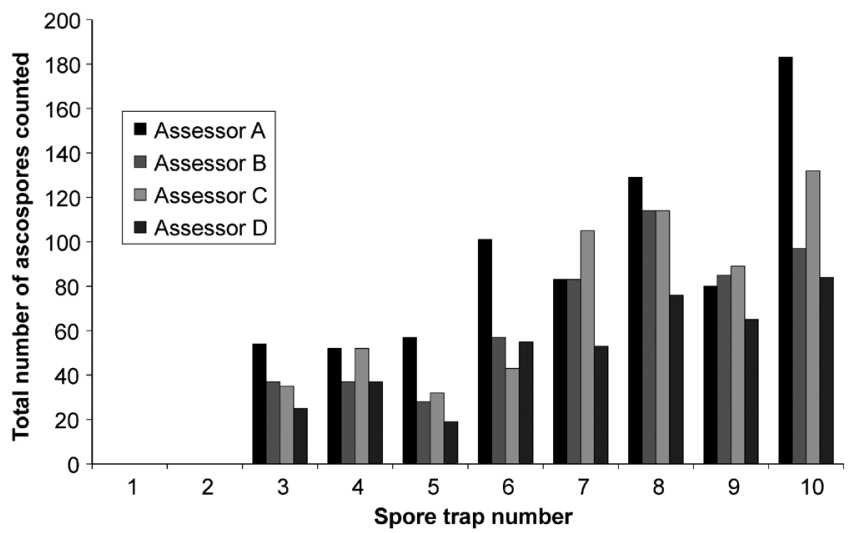

FIGURE 1: Total number of $N$. fuckeliana ascospores per slide counted by each assessor for ten spore traps. Assessors randomly selected five (out of a possible 10) transects to count on each slide. 


\section{Examining single transects}

A significant level of variation was also observed between assessors when examining exactly the same transects of slides (Fig. 2, $\mathrm{P}=0.0003$ ). The difference between the highest and lowest assessor ascospore count for a single transect ranged from 6 ascospores for transect 1 through to 33 ascospores for transect 4 . Assessor D (18 \pm 1 spores/slide) was consistently lower than all other assessors $(26 \pm 2,26 \pm 2$ and $23 \pm 2$ spores/slide for Assessors A, B and C respectively), who did not differ significantly from each other. In this experiment, there was no significant difference between the number of grouped or singleton ascospores counted by each assessor ( $\mathrm{P}=0.1395$ and $\mathrm{P}=0.0711$ respectively). For the single transects, grouped ascospores accounted for an average of $50 \%$ of the total spores counted although this was highly variable between assessors and spores traps (range 0-88\%).

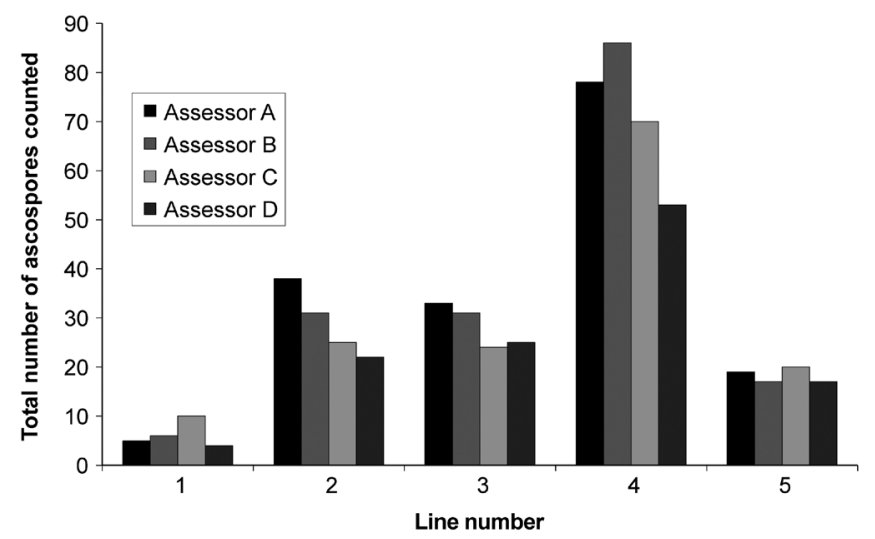

FIGURE 2: Total number of $N$. fuckeliana ascospores per transect counted by each assessor for the same five single transect views from one spore trap.

\section{DISCUSSION}

In this study, a high level of variation in ascospores numbers was found between assessors for the same slides. As N. fuckeliana ascospores are sticky and dispersed by water (P.E. Crane, Scion, unpublished data) they are likely to have a highly clumped/ variable distribution across the slide. As only one half of a slide is counted during the standard counting process each time a slide is examined, even by the same assessor, it is likely that a slightly different area of the slide will be examined and thus a different count obtained. However, a very wide range of variation in ascospore numbers was observed in this study. This, combined with the consistent patterns of counting demonstrated by some assessors (e.g. Assessor D consistently lower than all other assessors) and the highly variable results of the single transect counts, indicates that there are likely to be other sources of assessor variation present.

There are a number of possible sources of variation in this study. Of the three types of errors outlined by Elphick (2008), both type two and type three errors are probably contributing to the variation observed in the present study. First, it is probable that some of the variation in $N$. fuckeliana ascospore numbers was due to detection mistakes, a type two error (Elphick 2008). Correct counting in this study required finding the $N$. fuckeliana ascospores on the slide surface. Although the ascospores never appeared to be hidden by other spores (and thus not detectable), they often stained poorly or lost their stain over time. In these cases, ascospores could be seen only through careful slide 
scanning or by slight vertical movement of the slide to pick up light reflected from the guttules. Furthermore, ascospores were often observed within clumps of other spores at the edge of water droplets, making detection more difficult. When $N$. fuckeliana ascospores were found on the edge of the microscope field of view, they were also sometimes difficult to visualise.

Variation in counts of ascospores is also likely to be due to individual spores being misidentified. This is a type three error: erroneous counts (Elphick 2008). Although $N$. fuckeliana ascospores are fairly distinctive in appearance there can be slight variation in size and shape, which some assessors may not have recognised. In addition, a number of other two-celled spores were observed on spore traps and these may have been confused with $N$. fuckeliana ascospores by some assessors and included in the count.

One encouraging outcome of the calibration of spore counting is that none of the assessors recorded any $N$. fuckeliana ascospores on two of the slides. From this it appears likely that slides with no ascospores will be correctly counted and in this instance no assessor misidentified other spores as $N$. fuckeliana ascospores.

A number of techniques could be used to improve counts in this study. Training of assessors may improve their recognition of ascospores and provide more consistent spore counts. This may not remove all variation, however, as there is a level of subjectivity, although small, in spore identification. For example, Alldredge et al. (2008) found that further training did not remove all observer differences: substantial differences in avian point counts were found between paired observers even when trials involved highly experienced field workers. Clearly, assessor bias can be avoided by using a single assessor to count all spore traps, but this may not be practical in some situations. Another solution, if absolute spore numbers are required, could be to use a multiple observer/ assessor technique as advocated by Jenkins \& Manly (2008) and others (see Elphick 2008). These studies have recommended using an average of the counts of two or more assessors to give more consistent spore numbers.

More consistency in counts could be gained by manipulating the data after counting. In situations where one or two assessors are consistently higher or lower than the others, their counts could be multiplied by a constant to align them more closely with the main group of assessors. In the current study, for example, all counts from Assessor D could be multiplied by 1.25 so that they align more closely with counts from the other assessors.

Alternatively, it is possible to account for assessor variation in the data by using data categories rather than absolute numbers. These categories could be ranges of ascospore numbers, which account for the some of the range of variation in counts between assessors. Thus spore traps could be assessed as having 0 spores, $<10$ spores, 10-50 spores, 50-100 spores and so on. The development of these ranges would depend on the amount of variation present between assessors and the range of categories that would provide useful data for the study at hand.

A completely different approach to quantifying spores would be to move away from manual assessment using a microscope to molecular techniques, which have been used to quantify spores of specific fungal pathogens in environmental samples (Zeng et al. 2004) and spore traps (Schweigkofler et al. 2004). Molecular detection methods (i.e. species specific nested-PCR) have been developed for N. fuckeliana (Langrell 2004), and could be calibrated for quantification of spore traps.

This calibration study has demonstrated that there is considerable assessor variation in ascospore counts of $N$. fuckeliana spore traps. How this variation is reduced in the methodology, or dealt with in final analyses, depends on the end use of the data and on whether it is important to obtain absolute spore numbers for the study. This calibration exercise has also served as an appropriate reminder of the importance of understanding the limitations of collected data prior to analyses. 


\section{ACKNOWLEDGEMENTS}

Thanks to Judy Gardner and Rebecca Ganley who took part in the spore calibration exercise. Pat Crane was responsible for setting up the spore trapping initially and the traps were maintained by City Forests Ltd staff, particularly Ross Chambers. Mark Kimberley undertook the statistical analyses, and John Bain and Rob Beresford provided useful feedback on an earlier draft of this manuscript. Funding was provided by the Foundation for Research, Science and Technology (CO4X0807), the Forest Biosecurity Research Council and the Nectria Focus Group.

\section{REFERENCES}

Alldredge MW, Pacifici K, Simons TR, Pollock KH 2008. A novel field evaluation of the effectiveness of distance sampling and double independent observer methods to estimate aural avian detection probabilities. Journal of Applied Ecology 45: 1349-1356.

Amsalem L, Freeman S, Rav-David D, Nitzani Y, Sztejnberg A, Pertot I, Elad Y 2006. Effect of climatic factors on powdery mildew caused by Sphaerotheca macularis f.sp. fragariae on strawberry. European Journal of Plant Pathology 114: 283-292.

Dick MA, Crane PE 2009. Neonectria fuckeliana is pathogenic to Pinus radiata in New Zealand Australasian Plant Disease Notes 4: 12-14.

Elphick CS 2008. Guest Editorial. How you count counts: the importance of methods research in applied ecology. Journal of Applied Ecology 45: 1313-1320.

Gadgil PD, Dick MA, Dobbie K 2003. Fungi Silvicolae Novazelandiae: 4. New Zealand Journal of Forestry Science 33: 265-272.

Geagea L, Huber L, Sache I, Flura D, McCartney HA, Fitt BDL 2000. Influence of simulated rain on dispersal of rust spores from infected wheat seedlings. Agricultural and Forest Meteorology 101: 53-66.

Greer CA, Webster RK 2001. Occurrence, distribution, epidemiology, cultivar reaction, and management of rice blast disease in California. Plant Disease 85: 1096-1102.

Ingold CT 1953. Dispersal in Fungi. Clarendon Press, Oxford. 197 p.

Jenkins KJ, Manly BFL 2008. A double-observer method for reducing bias in faecal pellet surveys of forest ungulates: Roosevelt elk and Columbian black-tailed deer in Olympic National Park, USA. Journal of Applied Ecology 45: 1339-1348.

Langrell SRH 2004. Development of a nested PCR detection procedure for Nectria fuckeliana direct from Norway spruce bark extracts. FEMS Mircobiology Letters 242: 185-193.

Ostry ME, Nicholls TH 1982. A technique for trapping fungal spores. USDA Forest Service, North Central Forest Experimental Station. Research Note NC-283.

Schweigkofler W, O'Donnell K, Garbelotto M 2004. Detection and quantification of airborne conidia of Fusarium circinatum, the causal agent of Pine Pitch Canker, from two California sites by using a real-time PCR approach combined with a simple spore trapping method. Applied and Environmental Microbiology 70: 3512-3520.

Vasiliauskas R, Stenlid J 1997. Population structure and genetic variation in Nectria fuckeliana. Canadian Journal of Botany 75: 1707-1713.

Zeng Q-Y, Westermark S-O, Rasmuson-Lestander A, Wang X-R 2004. Detection and quantification of Wallemia sebi in aerosols by real-time PCR, conventional PCR and cultivation. Applied and Environmental Microbiology 70: 7295-7302. 